\title{
Nota Terapéutica: Las implicancias clínicas de importantes ensayos terapéuticos recientes
}

\begin{abstract}
Clinical implications of recent key therapeutic trials. Letter, issue 65, July - August 2007. Traducido con autorización de "Therapeutics Initiative" (The University of British
\end{abstract} Columbia). Disponible en URL: http://www.ti.ubc.ca/PDF/65.pdf

\begin{abstract}
Los ensayos clínicos aleatorizados son factores críticos para determinar el efecto neto de una intervención sanitaria (diferencia entre beneficios y daños)
\end{abstract}

Cuando el resultado principal de un ensayo clínico (ECA) es la reducción de uno o más eventos adversos serios, la mejor medida del efecto sanitario neto de la medicación es el número total de personas que sufrieron al menos un evento adverso serio (ver la carta terapéutica 42). Cuando este número no se reporta, la mortalidad total se convierte en la mejor medida del efecto sanitario neto. En esta carta terapéutica hemos utilizado esta perspectiva analítica para interpretar los resultados y las implicancias clínicas de cuatro ECA recientes.

\section{Torcetrapib para la prevención de eventos cardiovasculares}

El estudio de investigación del manejo del nivel lipídico y su impacto en los eventos relacionados con la arterioesclerosis (ILLUMINATE) no ha sido aún publicado. Este estudio fue diseñado con el fin de evaluar si la nueva droga torcetrapib, un inhibidor de la proteína transportadora de colesterol, reducía la mortalidad y morbilidad en relación al placebo.

En estudios previos el torcetrapib, a una dosis de 30 a 90 $\mathrm{mg} /$ día, había provocado un aumento del HDL relacionado con la dosis, de hasta un $55 \%$, así como una disminución del LDL, tambien relacionada con la dosis, de hasta un $19 \%^{12}$. Los investigadores del ILLUMINATE aleatorizaron alrededor de 15000 pacientes de alto riesgo cardíaco a recibir atorvastatina o la combinación de atorvastatina más torcetrapib $60 \mathrm{mg}$, con la idea de seguir a los sujetos durante cuatro a cinco años.

El 6 de Diciembre de 2006, un poco después de cumplirse el primer año del estudio, Pfizer suspendió el ensayo y discontinuó el desarrollo del torcetrapib ${ }^{3}$. Esto se debió a la decisión del Comité de Seguridad y Monitoreo de datos del ensayo clínico, quien descubrió un aumento estadísticamente significativo en la mortalidad de los pacientes que recibían torcetrapib (82 muertes) en relación a los que recibían placebo (51 muertes). Este resultado se obtuvo a pesar del aumento del HDL y de la reducción del LDL que producía el torcetrapib.

\section{Implicancias clínicas}

El torcetrapib aumenta la mortalidad de los pacientes de alto riesgo vascular, comparado con placebo. Los cambios en puntos finales subrogantes -elevar el HDL y disminuir el LDL- que puede producir una droga, no son suficientes para conocer si la misma es beneficiosa o perjudicial.

\section{Comentario}

Este ensayo clínico arroja luz sobre cómo debería funcionar el sistema. El estudio comenzó durante una etapa relativamente temprana del desarrollo de la droga, ya que la Administración de Drogas y Medicamentos (en inglés FDA) iba a solicitar evidencia sobre la reducción de la mortalidad y la morbilidad antes de la aprobación del torcetrapib. El Comité de Seguridad y Monitoreo de datos del ensayo clínico hizo bien su trabajo al detectar y reportar el problema y la compañía farmacéutica respondió apropiadamente. Por lo tanto, se detectó relativamente temprano que la droga podía ser peligrosa y se minimizó la cantidad de pacientes expuestos y dañados por ella. Un punto crítico es que este estudio llegue a ser publicado y que se reporten todos los eventos adversos serios, para poder evaluar los posibles mecanismos de daño relacionados al torcetrapib.
Intervención coronaria percutánea (ICP) para la angina estable $^{4}$

En este ensayo clínico se aleatorizaron 2287 pacientes con isquemia miocárdica y enfermedad coronaria significativa a recibir intervención coronaria percutánea (ICP: 1145) o ninguna intervención (1138). Ambos grupos recibieron tratamiento farmacológico estándar basado en la evidencia (aspirina, estatinas, beta bloqueantes e inhibidores de la enzima convertidora de angiotensina) asociado a intervenciones sobre el estilo de vida. Luego de un seguimiento promedio de 4,6 años, la ICP no tuvo ningún efecto sobre el punto final primario: muerte por cualquier causa o infarto agudo de miocardio no fatal $19 \%$ en el grupo ICP vs. 18,5\% en el grupo control (HR 1,05; 0,87 a $1,27)$. Los puntos finales secundarios tampoco fueron diferentes entre los grupos. El grupo control experimentó más angina de pecho y recibió más nitratos (aumento de riesgo absoluto o ARA 14\%) y bloqueantes calcicos (ARA $8 \%$ ) que el grupo ICP. De todas maneras, a los cinco años de seguimiento el porcentaje de pacientes libres de angina no difirió en ambos grupos: $74 \%$ en el grupo ICP y $72 \%$ en el control. La mortalidad total, el único punto final reportado relacionado al beneficio sanitario neto, fue de $7,4 \%$ en el grupo ICP y de $8,3 \%$ en el grupo control: HR 0,87; 0,65 a 1,16.

\section{Implicancias clínicas}

Como estrategia de manejo inicial en pacientes con angina estable relacionada a enfermedad coronaria y comparada con la no intervención, la intervención coronaria percutánea no reduce la mortalidad ni los eventos adversos serios.

\section{Comentario}

El amplio intervalo de confianza relacionado a la mortalidad total deja una cierta duda en relación al beneficio sanitario neto. El reporte publicado en el New England Journal of Medicine hubiera sido más informativo si hubiera incluido el número total de individuos dentro de cada grupo con al menos un evento adverso serio.

\section{Acido eicosapentanoico para la hipercolesterolemia ${ }^{5}$}

El ácido eicosapentanoico (EPA) es un ácido graso ommega 3 de cadena larga presente en los aceites de pescado. En este ensayo clínico abierto, 18645 personas japonesas con un nivel de colesterol total mayor a $252 \mathrm{mg} / \mathrm{dL}$ fueron aleatorizadas a recibir $600 \mathrm{mg}$ de EPA tres veces por día o placebo. Estos pacientes fueron seguidos durante un promedio de 4,6 años. Ambos grupos fueron tratados con bajas dosis de estatinas (10 $\mathrm{mg} /$ día de pravastatina ó $5 \mathrm{mg} /$ día de sinvastatina). El ensayo utilizó puntos finales ciegos para reducir los posibles sesgos. Del total de pacientes aleatorizados, $20 \%$ eran de prevención secundaria y $80 \%$ de prevención primaria. El punto final primario compuesto (muerte súbita de orígen cardiovascular, infarto agudo de miocardio fatal o no fatal, angina inestable, angioplastia y colocación de stent o cirugía de revascularización coronaria ocurrió en el $2,8 \%$ del grupo EPA vs. en el 3,5 del grupo control (HR 0,81; 0,69 a 0,95) con una reducción de riesgo absoluto (RRA) de $0,7 \%$ y un número necesario a tratar (NNT) de 143 para prevenir un evento. La reducción absoluta de riesgo para el punto final primario fue del $2 \%$, estadísticamente significativa en el grupo de prevención secundaria $(p=0,048)$ y del $0,3 \%$ (no significativa) en el grupo de prevención primaria $(p=0,132)$. La tasa de abandono del tratamiento debido a eventos adversos fue del $11,7 \%$ para el 
grupo EPA vs. $7,2 \%$ en el grupo control, con un RR 1,6; 1,5 a 1,8 ; un aumento absoluto del riesgo (AAR) de $4,5 \%$ y un número necesario de pacientes para que uno desarrolle un daño durante cinco años o "número necesario para dañar" (NND) de 22. La mortalidad total, el único punto final relacionado con el efecto sanitario neto que fue reportado, fue de $3,1 \%$ para el grupo EPA y de 2,8\% para el grupo control, HR 1,09; 0,92 a 1,27 .

\section{Implicancias clínicas}

En una población japonesa el EPA no afecta la mortalidad (prevención primaria y secundaria). EI EPA reduce los eventos adversos cardiovasculares serios en poblaciones de prevención secundaria en relación al placebo.

\section{Comentario}

Este gran ensayo tiene la posibilidad de brindar una estimación precisa del efecto sanitario neto del EPA en una población de prevención primaria y secundaria. Lamentablemente, el reporte publicado en el Lancet no incluyó el número total de gente que sufrió al menos un evento adverso serio por cada grupo y subgrupo.

Anticoagulantes orales y antiplaquetarios para el tratamiento de la enfermedad vascular periférica ${ }^{6}$

Este ensayo clínico abierto aleatorizó 2161 pacientes con enfermedad arterial periférica de las extremidades inferiores, de las carotídas o de los vasos subclavios, a recibir tratamiento antiplaquetario solo o en combinación con anticoagulantes, durante un promedio de 2,9 años. Las drogas antiplaquetarias utilizadas fueron el ácido acetilsalicílico a una dosis de 81 a $325 \mathrm{mg} /$ día, clopidogrel o ticlopidina. Los anticoagulantes orales utilizados fueron warfarina o acenocoumarol.

El infarto agudo de miocardio, el accidente cerebro-vascular, la isquemia severa o la muerte de causa cardiovascular ocurrieron en el $15,9 \%$ de los pacientes del grupo de tratamiento combinado y en el $17,4 \%$ del grupo de tratamiento antiplaquetario, RR 0,$91 ; 0,74$ a 1,12 . Los sangrados con riesgo de muerte ocurrieron en el $4 \%$ de los pacientes del grupo combinación y en el $1,2 \%$ de los del grupo "tratamiento antiplaquetario" RR $3,4(1,8$ a 6,4$)$ AAR $2,8 \%$ y NND 36 durante tres años. La mortalidad total, el único efecto sanitario neto reportado, fue de $9,2 \%$ para el grupo combinación vs. $8,9 \%$ para el grupo antiplaquetario, RR $1,04(0,79$ a 1,38$)$.

\section{Implicancias clínicas}

En los pacientes con enfermedad vascular periférica y comparando esta estrategia con el tratamiento antiplaquetario; el agregado de un anticoagulante oral aumenta los episodios de sangrado con riesgo de muerte sin afectar mortalidad total o los eventos adversos cardiovasculares serios.

\section{Comentario}

Una vez más, el reporte del número total de gente con al menos un evento adverso serio en cada grupo hubiera aportado una medida más precisa del efecto sanitario neto.

Esta Carta Terpéutica fue remitida para su revisión a 40 expertos y médicos de atención primaria para poder corregir cualquier inexactitud y asegurar que la información sea concisa y relevante para los médicos.

Ver glosario*

\section{Referencias}

1. Davidson MH, McKenney JM, Shear CL, Revkin JH. Efficacy and safety of torcetrapib, a novel cholesteryl ester transfer protein inhibitor, in individuals with below-average high-density lipoprotein chlolesterol levels. J Am Coll Cardiol. 2006;48:1774-81.

2. McKenney JM, Davidson MH, Shear CL, Revkin JH. Efficacy and safety of torcetrapib, a novel cholesteryl ester transfer protein inhibitor, in individuals with below-average high-density lipoprotein cholesterol levels on a background of atorvastatin. J Am Coll Cardiol. 2006;48:1782-90.

3. Tall AR. CETP inhibitors to increase HDL cholesterol levels. N Engl J Med. 2007;356:1364-1366.

4. Boden WE, O'Rourke RA, Teo KK, el al. COURAGE Trial Research Group. Optimal medical therapy with or without PCl for stable coronary disease. N Engl J Med. 2007;356:1503-16.

5. Yokoyama M, Origasa H, Matsuzaki M,et al. Japan EPA lipid intervention study (JELIS) Investigators. Effects of eicosapentaenoic acid on major coronary events in hypercholesterolaemic patients (JELIS): a randomised openlabel,blinded endpoint analysis. Lancet. 2007;369:1090-8.

6. The Warfarin Antiplatelet Vascular Evaluation (WAVE) Trial Investigators, Anand S, Yusuf S, Xie C, et al. Oral anticoagulant and antiplatelet therapy and peripheral arterial disease. N Engl J Med. 2007;357:217-27. 